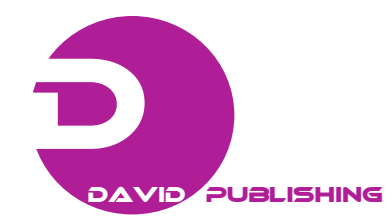

\title{
Effective Food Safety Audit for Restaurants
}

\author{
Yumiko Hirai \\ Osaka Seikei College, Matsukage Ookubo-cho, Akashi-City, Hyogo 1119-4-104, Japan \\ *Corresponding author's e-mail: yumi.pingjing@gmail.com
}

\begin{abstract}
The basics of food hygiene is hand washing. This is an undoubted fact, however, upon restaurant audits, ratio of issues related to food control and kitchen equipment management is much higher than personal hygiene as hand washing, and moreover, its improvement measures require a long time in reality. Therefore, even if personnel keep a rule of personal hygiene such as hand washing, food control and kitchen equipment management issues may cause food borne illness. To perceive the food borne illness possibility, specific audit items and timing becomes the key. In other words, when, where, and how should the audit take place? Audit results change in accordance to the criteria. In order to fully understand the audited organization's current situation, authors need to define the exact timing and audit items to address the audited organization in question. The findings from the restaurant audits and guidance are as follows: Audit is preferred during the actual working hours; Key audit point for food control is to check the existence of cross contamination and food temperature; Key audit point for kitchen equipment is 5S, especially on cleaning and washing; Audit items should be customized accordingly to the target; The same audit format should not be applied under a restaurant category.
\end{abstract}

Key words: Food hygiene audit, hand washing, restaurant audits, food control, kitchen equipment management. 